\title{
Elliptical rotation of a bosonic oscillator in ultrastrong waveguide QED
}

\author{
Kazuki Koshino ${ }^{0}$ \\ College of Liberal Arts and Sciences, Tokyo Medical and Dental University, Ichikawa, Chiba 272-0827, Japan
}

(Received 8 January 2021; accepted 5 April 2021; published 19 April 2021)

\begin{abstract}
We investigate the optical response of a linear waveguide quantum electrodynamics (QED) system, namely, a bosonic oscillator coupled to a waveguide. Our analysis is based on exact diagonalization of the overall Hamiltonian and is therefore rigorous even in the ultrastrong-coupling regime of waveguide QED. Owing to the counter-rotating terms in the oscillator-waveguide coupling, the phase-space motion of the oscillator is elliptical in general, and this becomes remarkable in the ultrastrong-coupling regime. We also reveal that such elliptical motion does not propagate into the output field and present an analytic form of the reflection coefficient that is asymmetric with respect to the resonance frequency.
\end{abstract}

DOI: 10.1103/PhysRevResearch.3.023060

\section{INTRODUCTION}

Cavity quantum electrodynamics (QED) deals with the interaction between a single atom and a discretized photon mode confined in a resonator, which is the simplest embodiment of quantum light-matter interaction. The cavity QED systems have been realized in various physical platforms: just to cite a few, single atoms coupled to an optical cavity, a semiconductor quantum dot in a photonic-crystal cavity, and a superconducting qubit coupled to a transmission-line resonator. Interestingly, regardless of its physical platform, a cavity QED system is characterized by several universal parameters, such as $\omega_{a}$ and $\omega_{c}$ (atom and cavity frequencies), $g$ (atom-photon coupling), $\kappa$ (cavity decay rate), and $\gamma$ (atomic decay rate into environments). In the history of cavity QED, extensive efforts have been made to reach the strong-coupling regime $(g>\kappa, \gamma)$, where the vacuum Rabi oscillation and splitting become observable [1-4]. In usual strong-coupling systems, the coupling is still by far smaller than the resonance frequencies of the atom and cavity. Recently, attainments of the ultrastrong-coupling $\left(g \gtrsim \omega_{a, c} / 10\right)$ and deep-strongcoupling $\left(g \gtrsim \omega_{a, c}\right)$ regimes have been reported [5-12]. In such ultrastrong-coupling systems, the counter-rotating terms in the Hamiltonian, which do not conserve the total number of excitations and are usually negligible in the weakly coupled systems, result in several intriguing physical phenomena, such as the Bloch-Siegert shift $[13,14]$, virtual photons in the ground state [15-19], and multiphoton vacuum Rabi oscillation [20-22].

Waveguide QED deals with the interaction between a single atom and a one-dimensional continuum of photon modes, typically provided by a waveguide attached to the atom. The parameters to characterize waveguide QED systems are $\omega_{a}, \gamma_{e}$ (atomic decay rate into waveguide), and $\gamma_{i}$ (atomic decay rate

Published by the American Physical Society under the terms of the Creative Commons Attribution 4.0 International license. Further distribution of this work must maintain attribution to the author(s) and the published article's title, journal citation, and DOI. into environments). The strong-coupling regime in waveguide QED is defined by $\gamma_{e} \gtrsim \gamma_{i}$, namely, the condition that radiation from the atom is dominantly forwarded to the waveguide [23-28]. This is reflected in spectroscopy as a strong suppression of transmission near the atomic resonance. Following cavity QED, the ultrastrong waveguide QED is defined as $\gamma_{e} \gtrsim \omega_{a} / 10$ [29] and the deep-strong waveguide QED should be defined as $\gamma_{e} \gtrsim \omega_{a}$. These regimes of waveguide QED have already been reached using a superconducting qubit [29,30]. Theoretically, up to the usual strong-coupling regime, perturbative treatment of dissipation based on the rotating-wave and Born-Markov approximations provides convenient and powerful theoretical tools, such as the Lindblad master equation and the input-output formalism [31,32]. However, this is not the case in highly dissipative regimes, and rigorous numerical methods are actively developed [33-36].

In this study, we investigate a linear waveguide QED setup, namely, a bosonic oscillator coupled to a waveguide, and investigate its optical response to a classical drive field applied through this waveguide. A merit of this system is that the overall Hamiltonian is diagonalizable by the Fano's method [37-39] and rigorous optical response is accessible even for highly dissipative situations. We report an elliptic phasespace motion of the oscillator due to the counter-rotating terms, which becomes remarkable in the ultrastrong-coupling regime. However, in contrast with the intuition provided by the input-output theory, such elliptic motion does not propagate into the waveguide. We also obtain an analytic formula of the reflection/transmission coefficient, which is asymmetric with respect to the dressed oscillator frequency. We hope that the rigorous optical response presented here would be useful for developing theoretical tools applicable to highly dissipative cavity and waveguide QEDs.

\section{THEORETICAL MODEL}

\section{A. Lagrangian to Hamiltonian}

In a setup considered in this study (Fig. 1), a bosonic oscillator is coupled to a semi-infinite waveguide and a monochromatic drive field is applied through this waveguide. 


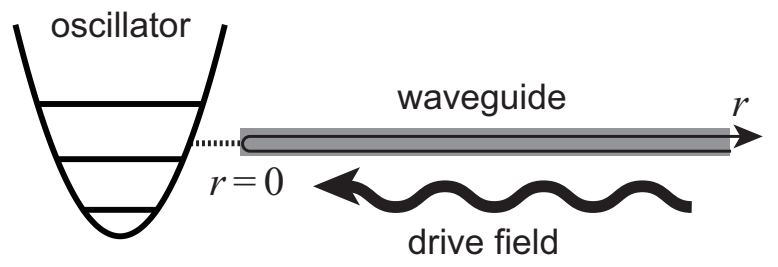

FIG. 1. Schematic of an oscillator-waveguide system. A bosonic oscillator is coupled to a semi-infinite waveguide, through which a monochromatic drive field is applied. The $r<0(r>0)$ region in the waveguide corresponds to the input (output) port.

We denote the bare resonance frequency and the amplitude of the oscillator by $\omega_{0}$ and $x$, respectively. The waveguide modes are labeled by a continuous wave vector $k$. We denote the frequency and the amplitude of the waveguide mode $k$ by $\omega_{k}$ and $x_{k}$, respectively. Assuming a linear dispersion $\omega_{k}=v k$, where $v$ is the velocity of the waveguide photon, the classical Lagrangian of this system is given by ( $v=1$ for simplicity)

$$
L=\frac{\dot{x}^{2}-\omega_{0}^{2} x^{2}}{2}+\int_{0}^{\infty} d k \frac{\dot{x}_{k}^{2}-k^{2} x_{k}^{2}}{2}+\int_{0}^{\infty} d k \eta_{k} x \dot{x}_{k},
$$

where the oscillator-waveguide coupling $\eta_{k}$ is real. The conjugate momenta corresponding to $x$ and $x_{k}$ are $p=\partial L / \partial \dot{x}=\dot{x}$ and $p_{k}=\partial L / \partial \dot{x}_{k}=\dot{x}_{k}+\eta_{k} x$. After the Legendre transformation, $H=\dot{x} p+\int_{0}^{\infty} d k \dot{x}_{k} p_{k}-L$, the classical Hamiltonian is given by

$$
H=\frac{p^{2}+\omega_{1}^{2} x^{2}}{2}+\int_{0}^{\infty} d k \frac{p_{k}^{2}+k^{2} x_{k}^{2}}{2}-\int_{0}^{\infty} d k \eta_{k} x p_{k},
$$

where the renormalized oscillator frequency $\omega_{1}$ is given by

$$
\omega_{1}^{2}=\omega_{0}^{2}+\int_{0}^{\infty} d k \eta_{k}^{2}
$$

Quantization is done by imposing the commutation relations, $[\hat{x}, \hat{p}]=i$ and $\left[\hat{x}_{k}, \hat{p}_{k^{\prime}}\right]=i \delta\left(k-k^{\prime}\right)(\hbar=1$ for simplicity). We introduce the annihilation operators by $\hat{b}=\left(\omega_{1} \hat{x}+\right.$ $i \hat{p}) / \sqrt{2 \omega_{1}}$ and $\hat{c}_{k}=\left(i k \hat{x}_{k}-\hat{p}_{k}\right) / \sqrt{2 k}$. The commutators for these operators are $\left[\hat{b}, \hat{b}^{\dagger}\right]=1$ and $\left[\hat{c}_{k}, \hat{c}_{k^{\prime}}^{\dagger}\right]=\delta\left(k-k^{\prime}\right)$, respectively. The quantized Hamiltonian is given by

$$
\begin{gathered}
\hat{H}=\omega_{1} \hat{b}^{\dagger} \hat{b}+\int_{0}^{\infty} d k\left[k \hat{c}_{k}^{\dagger} \hat{c}_{k}+\xi_{k}\left(\hat{b}^{\dagger}+\hat{b}\right)\left(\hat{c}_{k}^{\dagger}+\hat{c}_{k}\right)\right], \\
\xi_{k}=\frac{\eta_{k}}{2} \sqrt{\frac{k}{\omega_{1}}} .
\end{gathered}
$$

In this study, in order to apply the Fano diagonalization, we assume the following conditions on the coupling constants [38]: (i) $\eta_{k}\left(\xi_{k}\right)$ is nonzero for $k>0$ and (ii) $\eta_{k}^{2}\left(\xi_{k}^{2}\right)$ is a even (odd) function of $k$.

\section{B. Drude coupling}

To be more concrete, we assume that the coupling $\eta_{k}$ is insensitive to $k$ in the relevant frequency region,

$$
\eta_{k}^{2}=\frac{2 f / \pi}{1+\left(k / \omega_{x}\right)^{2}},
$$

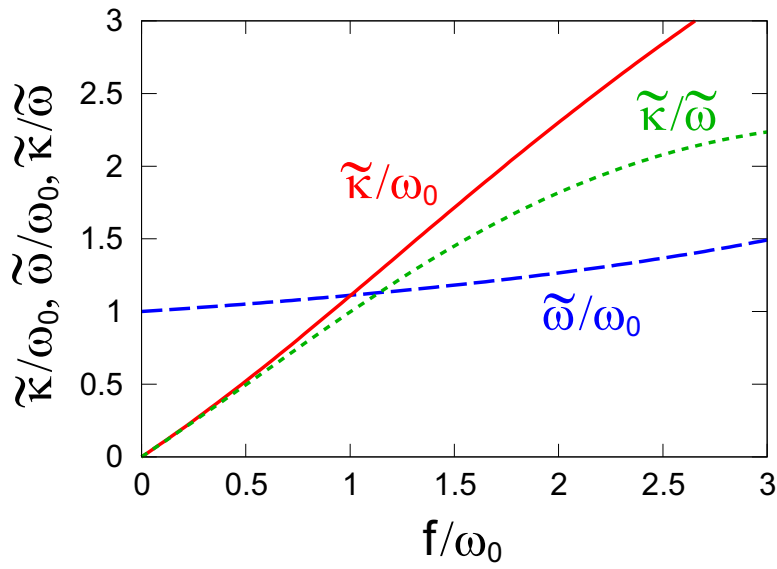

FIG. 2. Dependences of the linewidth $\widetilde{\kappa}$ (solid) and dressed resonance frequency $\widetilde{\omega}$ (dashed) of the oscillator on the coupling strength $f$. Their ratio, $\widetilde{\kappa} / \widetilde{\omega}$, is plotted by a dotted line. $\omega_{x}=5 \omega_{0}$. The ultrastrong coupling $(\tilde{\kappa} / \widetilde{\omega}>0.1)$ is attained for $f / \omega_{0}>0.103$ and the deep-strong coupling $(\widetilde{\kappa} / \widetilde{\omega}>1)$ is attained for $f / \omega_{0}>1.004$.

where $\omega_{x}$ is a cutoff frequency and $f$ is a constant that determines the coupling strength. From Eq. (3), $\omega_{1}$ is given by

$$
\omega_{1}=\sqrt{\omega_{0}^{2}+f \omega_{x}} .
$$

It is of note that, although $\omega_{1}$ diverges in the $\omega_{x} \rightarrow \infty$ limit, observable physical quantities such as the oscillator amplitude remain finite even in this limit (see Appendix E). $\xi_{k}$ is reduced to the following Drude form:

$$
\xi_{k}^{2}=\frac{f \omega_{x}^{2}}{2 \pi \omega_{1}} \frac{k}{k^{2}+\omega_{x}^{2}} .
$$

Note that, in circuit QED, the atom/cavity-waveguide coupling takes the Drude form [40]. We assume $\omega_{x} \gg \omega_{0}$ so that the coupling is Ohmic $\left(\xi_{k}^{2} \propto k\right)$ near the cavity resonance. We set $\omega_{x}=5 \omega_{0}$ hereafter, and observe the effects of cutoff frequency in Appendix E. As we observe in Fig. 2, $f$ is almost identical to the linewidth $\widetilde{\kappa}$ of the oscillator, particularly for weaker coupling. In this paper, we employ a dimensionless quantity $f / \omega_{0}$ as a measure of coupling strength.

\section{Linewidth and dressed frequency}

As the oscillator-waveguide coupling increases, the linewidth $\widetilde{\kappa}$ of the oscillator increases. Furthermore, the dressed resonance frequency $\widetilde{\omega}$ also changes from its bare value $\omega_{0}$ by renormalization. We determine these quantities through the phase shift upon reflection (Fig. 7). The dressed resonance is identified as the drive frequency achieving the $\pi$ phase shift, and the linewidth is identified as the difference between the drive frequencies achieving the $3 \pi / 2$ and $\pi / 2$ phase shifts. In terms of the transmissivity (Fig. 8), $\widetilde{\omega}$ and $\widetilde{\kappa}$ correspond to the center frequency of the dip and the full width at half maximum, respectively.

In Fig. 2, we plot the dependencies of $\widetilde{\kappa}$ and $\widetilde{\omega}$ on the coupling strength $f$. We observe that $\widetilde{\kappa}$ is almost identical to $f$, while $\widetilde{\omega}$ is not so sensitive to $f$. The ultrastrongcoupling regime $(\widetilde{\kappa} / \widetilde{\omega}>0.1)$ is achieved for $f / \omega_{0}>0.103$ and the deep-strong-coupling regime $(\widetilde{\kappa} / \widetilde{\omega}>1)$ is achieved 
$f / \omega_{0}>1.004$. In particular, in the weak-coupling region, $\widetilde{\omega}$ and $\widetilde{\kappa}$ are related to the complex frequency $\lambda_{1}$ of the oscillator as $\widetilde{\omega}=\operatorname{Re}\left(\lambda_{1}\right)$ and $\widetilde{\kappa} / 2=\operatorname{Im}\left(\lambda_{1}\right)$. Using the perturbative solution of $\lambda_{1}$ [Eq. (26)], we obtain $\widetilde{\omega} \approx \omega_{0}+\omega_{0} f / 2 \omega_{x}$ and $\widetilde{\kappa} \approx f$

\section{Initial state vector}

In this study, we investigate the optical response of the oscillator driven by a monochromatic classical field applied through the waveguide (Fig. 1). The positively rotating part of drive amplitude is given by

$$
E(r, t)=E_{d} e^{i k_{d}(r-t)},
$$

where $E_{d}$ and $k_{d}$ are the complex amplitude and wave number/frequency of the drive, respectively. At the initial moment $(t=0)$, we assume that the whole system is in the ground state except the drive field in the waveguide, which is in a coherent state. The initial state vector is then written as

$$
\left|\psi_{i}\right\rangle=\exp \left(\sqrt{2 \pi} E_{d} \hat{c}_{k_{d}}^{\dagger}-\sqrt{2 \pi} E_{d}^{*} \hat{c}_{k_{d}}\right)|\mathrm{vac}\rangle,
$$

where $|v a c\rangle$ is the ground state of the coupled system.

The real-space representation $\widetilde{c}_{r}$ of the waveguide field operator is defined as the Fourier transform of $\hat{c}_{k}$,

$$
\widetilde{c}_{r}=\frac{1}{\sqrt{2 \pi}} \int_{0}^{\infty} d k e^{i k r} \hat{c}_{k} .
$$

We can check that $\left\langle\widetilde{c}_{r}(0)\right\rangle \equiv\left\langle\psi_{i}\left|\widetilde{c}_{r}(0)\right| \psi_{i}\right\rangle=E(r, 0)$. Note that under this initial condition the drive field exists not only in the input port $(r<0)$ but also in the output port $(r>0)$. The latter propagates freely into positive direction without interacting with the oscillator.

Strictly speaking, the real-space representation of the waveguide mode depends on the boundary condition of the waveguide at $r=0$. For example, for a closed boundary condition, the waveguide mode function takes the form of $f_{k}(r)=\sqrt{2 / \pi} \sin (k r)=\left(i e^{-i k r}-i e^{i k r}\right) / \sqrt{2 \pi}$ [41]. Therefore, we should add a phase factor $i(-i)$ for the input (output) port in Eq. (11), which accounts for the sign flip upon reflection at a mirror. However, we employ Eq. (11) as the real-space representation of waveguide modes for simplicity. This introduces no problem except for definition of the relative phase in the input and output ports.

\section{DIAGONALIZATION}

\section{A. General formula}

The Hamiltonian [Eq. (4)] is bilinear in bosonic operators and can be diagonalized by the Fano's method. We can rewrite the Hamiltonian as

$$
\hat{H}=\int_{0}^{\infty} d k k \hat{d}_{k}^{\dagger} \hat{d}_{k}
$$

where $\hat{d}_{k}$ is an eigenmode annihilation operator satisfying the bosonic commutation relation,

$$
\left[\hat{d}_{k}, \hat{d}_{k^{\prime}}^{\dagger}\right]=\delta\left(k-k^{\prime}\right) .
$$

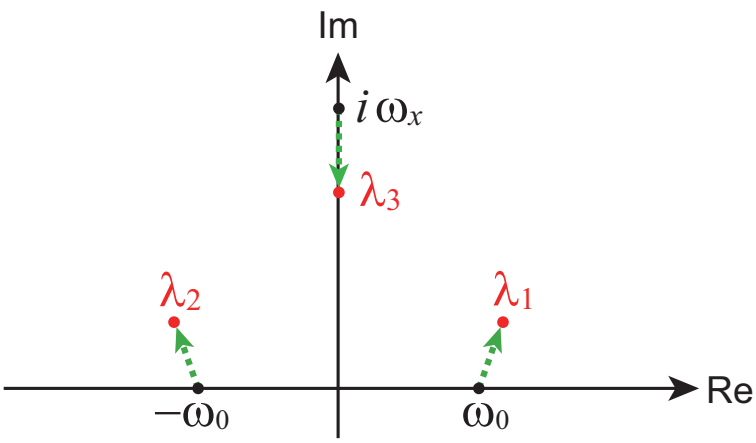

FIG. 3. $\lambda_{1,2,3}$ on the complex plane. Arrows indicate the directions as the coupling strength $f$ is increased.

$\hat{d}_{k}$ is given by linear combination of the original bosonic operators as

$$
\begin{aligned}
\hat{d}_{k}= & \beta_{1}(k) \hat{b}+\beta_{2}(k) \hat{b}^{\dagger} \\
& +\int_{0}^{\infty} d q\left[\gamma_{1}(k, q) \hat{c}_{q}+\gamma_{2}(k, q) \hat{c}_{q}^{\dagger}\right],
\end{aligned}
$$

where the coefficients are given by (see Appendix A for derivation)

$$
\begin{gathered}
\beta_{1}(k)=\frac{\left(k+\omega_{1}\right) \xi_{k}}{k^{2}-\omega_{1}^{2} z(k)}, \\
\beta_{2}(k)=\frac{\left(k-\omega_{1}\right) \xi_{k}}{k^{2}-\omega_{1}^{2} z(k)}, \\
\gamma_{1}(k, q)=\delta(k-q)+\widetilde{\gamma}_{1}(k, q), \\
\gamma_{2}(k, q)=\frac{2 \omega_{1} \xi_{k} \xi_{q}}{(k+q)\left[k^{2}-\omega_{1}^{2} z(k)\right]},
\end{gathered}
$$

where

$$
\widetilde{\gamma}_{1}(k, q)=\frac{2 \omega_{1} \xi_{k} \xi_{q}}{(k-q-i 0)\left[k^{2}-\omega_{1}^{2} z(k)\right]},
$$

and $z(k)$ is a dimensionless quantity representing the selfenergy correction for the oscillator frequency,

$$
z(k)=1+\frac{2}{\omega_{1}} \int_{-\infty}^{\infty} d q \frac{\xi_{q}^{2}}{k-q-i 0} .
$$

Note that $z(-k)=z^{*}(k)$, which results from the property that $\xi_{-k}^{2}=-\xi_{k}^{2}$. Inversely, the bare operators $\hat{b}$ and $\hat{c}_{k}$ are expressed in terms of the eigenoperators by

$$
\begin{gathered}
\hat{b}=\int_{0}^{\infty} d q\left[\beta_{1}^{*}(q) \hat{d}_{q}-\beta_{2}(q) \hat{d}_{q}^{\dagger}\right], \\
\hat{c}_{k}=\int_{0}^{\infty} d q\left[\gamma_{1}^{*}(q, k) \hat{d}_{q}-\gamma_{2}(q, k) \hat{d}_{q}^{\dagger}\right] .
\end{gathered}
$$

\section{B. Specific results for Drude coupling}

When the coupling is given by Eq. (8), $z(k)$ and $k^{2}-$ $\omega_{1}^{2} z(k)$ are rewritten as follows:

$$
\begin{aligned}
z(k) & =1+\frac{i f \omega_{x}^{2}}{\omega_{1}^{2}\left(k-i \omega_{x}\right)}, \\
k^{2}-\omega_{1}^{2} z(k) & =\frac{\left(k-\lambda_{1}\right)\left(k-\lambda_{2}\right)\left(k-\lambda_{3}\right)}{k-i \omega_{x}},
\end{aligned}
$$



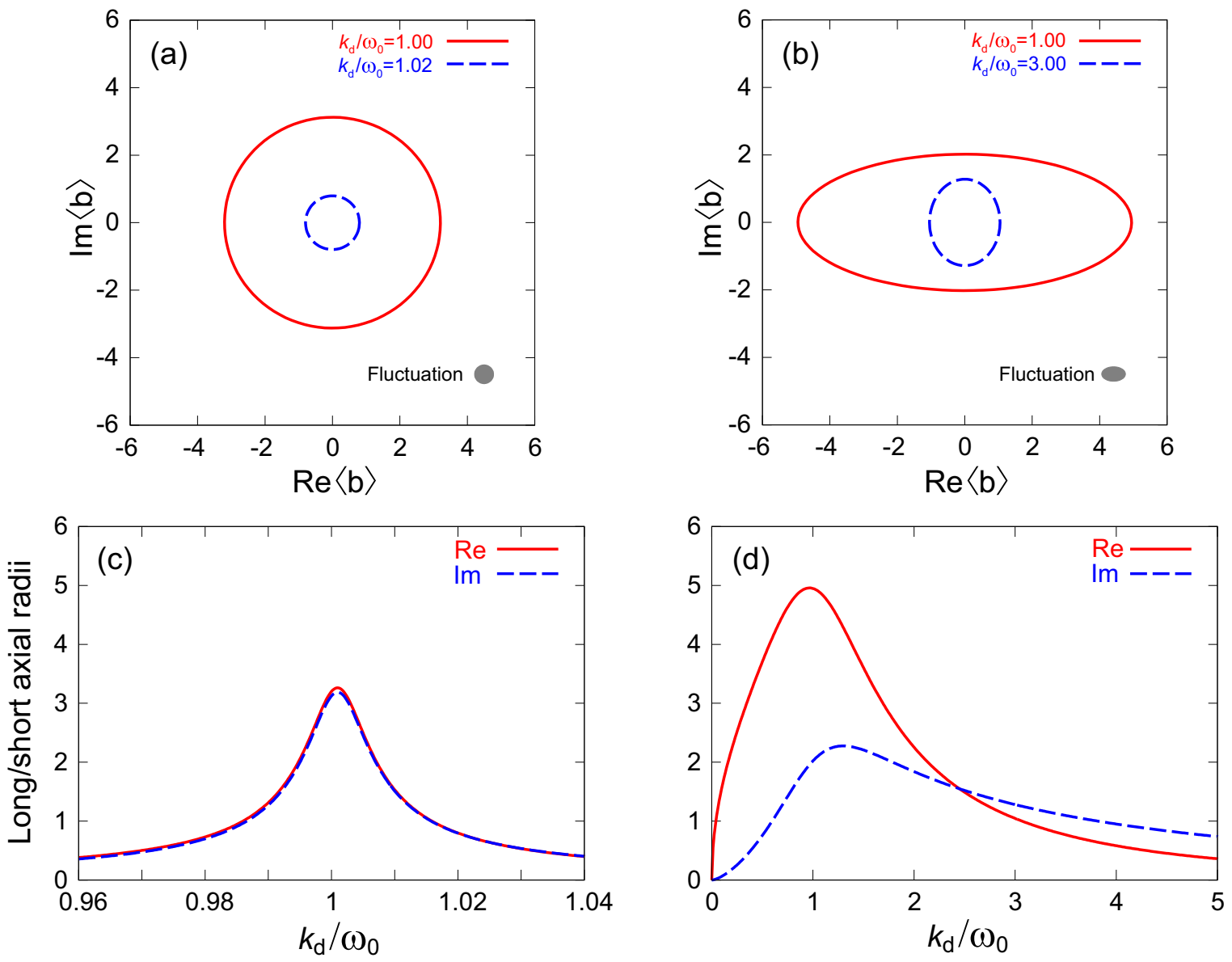

FIG. 4. Elliptical motion of the oscillator amplitude. (a) Trajectories on the phase space for $f / \omega_{0}=0.01$. The drive frequency is set at $k_{d}=1.00 \omega_{0}$ (resonance, solid) and $k_{d}=1.02 \omega_{0}$ (off resonance, dotted). The photon rate of the drive field is set at $\left|E_{d}\right|^{2}=2.5 \widetilde{\kappa}$, at which the mean oscillator excitation is roughly $\left\langle\hat{b}^{\dagger} \hat{b}\right\rangle=4\left|E_{d}\right|^{2} / \widetilde{\kappa}=10$ on resonance. The uncertainty ellipse is also shown. (b) The same plot as (a) for $f / \omega_{0}=1 . k_{d}=1.00 \omega_{0}$ (solid) and $k_{d}=3.00 \omega_{0}$ (dotted). (c) Dependence of the long and short axial radii on the drive frequency for $f / \omega_{0}=0.01$. (d) The same plot as (c) for $f / \omega_{0}=1$.

where $\lambda_{1,2,3}$ are the solutions of the following cubic equation for $k$,

$$
k^{3}-i \omega_{x} k^{2}-\omega_{1}^{2} k+i \omega_{x} \omega_{0}^{2}=0 .
$$

As shown in Fig. $3, \lambda_{1}\left(\lambda_{2}\right)$ is on the first (second) quadrant and $\lambda_{3}$ is on the positive imaginary axis. The real and imaginary parts of $\lambda_{1}$ correspond to the dressed resonance $\widetilde{\omega}$ and half of the linewidth $\widetilde{\kappa}$ unless the coupling is not very strong. For reference, we present the perturbative solution of Eq. (25) with respect to the coupling strength $f$. The zeroth-order solutions are $\lambda_{1}^{(0)}=\omega_{0}, \lambda_{2}^{(0)}=-\omega_{0}$, and $\lambda_{3}^{(0)}=i \omega_{x}$. Up to the first order in $f$, the three solutions are given by

$$
\lambda_{1} \approx\left(\omega_{0}+f \omega_{0} / 2 \omega_{x}\right)+i f / 2,
$$

$\lambda_{2} \approx-\left(\omega_{0}+f \omega_{0} / 2 \omega_{x}\right)+i f / 2$, and $\lambda_{3} \approx i \omega_{x}-i f$.

\section{OPTICAL RESPONSE}

\section{A. Oscillator amplitude}

In this section, we investigate time evolution of the whole system from the initial state vector, Eq. (10). We first observe the oscillator amplitude $\langle\hat{b}(t)\rangle \equiv\left\langle\psi_{i}|\hat{b}(t)| \psi_{i}\right\rangle$. Since $\hat{d}_{k}$ is an eigenoperator of the Hamiltonian, $\hat{b}(t)$ is given, from
Eq. (21), by

$$
\hat{b}(t)=\int_{0}^{\infty} d q\left[e^{-i q t} \beta_{1}^{*}(q) \hat{d}_{q}-e^{i q t} \beta_{2}(q) \hat{d}_{q}^{\dagger}\right] .
$$

Furthermore, $\left|\psi_{i}\right\rangle$ is an eigenstate of $\hat{d}_{q}$ and satisfies

$$
\hat{d}_{q}\left|\psi_{i}\right\rangle=\sqrt{2 \pi}\left[E_{d} \gamma_{1}\left(q, k_{d}\right)+E_{d}^{*} \gamma_{2}\left(q, k_{d}\right)\right]\left|\psi_{i}\right\rangle .
$$

From these results, $\langle\hat{b}(t)\rangle$ is given by

$$
\begin{aligned}
\langle\hat{b}(t)\rangle= & \sqrt{2 \pi} E_{d} \int_{0}^{\infty} d q\left[e^{-i q t} \beta_{1}^{*}(q) \gamma_{1}\left(q, k_{d}\right)\right. \\
& \left.-e^{i q t} \beta_{2}(q) \gamma_{2}^{*}\left(q, k_{d}\right)\right] \\
& +\sqrt{2 \pi} E_{d}^{*} \int_{0}^{\infty} d q\left[e^{-i q t} \beta_{1}^{*}(q) \gamma_{2}\left(q, k_{d}\right)\right. \\
& \left.-e^{i q t} \beta_{2}(q) \gamma_{1}^{*}\left(q, k_{d}\right)\right] .
\end{aligned}
$$

This is divided into stationary and transient components as $\langle\hat{b}(t)\rangle=\langle\hat{b}(t)\rangle_{s}+\langle\hat{b}(t)\rangle_{t}$. The stationary component is given by

$$
\langle\hat{b}(t)\rangle_{s}=\sqrt{2 \pi} \beta_{1}^{*}\left(k_{d}\right) E_{d} e^{-i k_{d} t}-\sqrt{2 \pi} \beta_{2}\left(k_{d}\right) E_{d}^{*} e^{i k_{d} t} .
$$


The transient component is presented in Appendix B. Putting $E_{d}=\left|E_{d}\right| e^{i \theta_{d}}$, we have

$$
\begin{aligned}
\operatorname{Re}\langle\hat{b}(t)\rangle_{s} & =\sqrt{8 \pi}\left|E_{d}\right| \omega_{1} \xi_{k_{d}} \operatorname{Re}\left(\frac{e^{i\left(k_{d} t-\theta_{d}\right)}}{k_{d}^{2}-\omega_{1}^{2} z\left(k_{d}\right)}\right), \\
\operatorname{Im}\langle\hat{b}(t)\rangle_{s} & =-\sqrt{8 \pi}\left|E_{d}\right| k_{d} \xi_{k_{d}} \operatorname{Im}\left(\frac{e^{i\left(k_{d} t-\theta_{d}\right)}}{k_{d}^{2}-\omega_{1}^{2} z\left(k_{d}\right)}\right) .
\end{aligned}
$$

These equations indicate that the phase-space motion of the oscillator amplitude $\langle\hat{b}(t)\rangle_{s}$ is elliptical in general. The ratio of the vertical (imaginary) radius relative to the horizontal (real) radius is $k_{d} / \omega_{1}$, and thus depends on the drive frequency. As a measure of the ellipticity of phase-space motion, we employ the ratio of the two axial radii at the dressed resonance,

$$
\mathcal{E}=\widetilde{\omega} / \omega_{1}
$$

As we observe in Figs. 4(a) and 4(c), such elliptical motion is not remarkable for weak coupling. Under the parameter of these figures, from Eqs. (48), (7), and (33), $\widetilde{\omega} / \omega_{0}=1.002$, $\omega_{1} / \omega_{0}=1.025$, and $\mathcal{E}=0.978$. In contrast, as we observe in Figs. 4(b) and 4(d), the phase-space motion becomes highly elliptical for stronger coupling. In these figures, $\widetilde{\omega} / \omega_{0}=$ $1.235, \omega_{1} / \omega_{0}=2.449$, and $\mathcal{E}=0.504$. These results agree with the general intuition that the counter-rotating terms, which are the origin of noncircular motion, become more crucial for stronger coupling.

\section{B. Quadrature fluctuations}

Here, we investigate the quadrature fluctuations of the oscillator. We define the $\hat{X}$ and $\hat{Y}$ quadratures by $\hat{X}=\left(\hat{b}+\hat{b}^{\dagger}\right) / 2$ and $\hat{Y}=-i\left(\hat{b}-\hat{b}^{\dagger}\right) / 2$, respectively, and their fluctuations by $\Delta X=\sqrt{\left\langle\hat{X}^{2}\right\rangle-\langle\hat{X}\rangle^{2}}$ and $\Delta Y=\sqrt{\left\langle\hat{Y}^{2}\right\rangle-\langle\hat{Y}\rangle^{2}}$, respectively, where $\langle\hat{O}\rangle=\left\langle\psi_{i}|\hat{O}| \psi_{i}\right\rangle$. From these definitions, we have

$$
\begin{aligned}
\Delta X & =\frac{\sqrt{1+2\left\langle\hat{b}^{\dagger}(t), \hat{b}(t)\right\rangle+2 \operatorname{Re}\langle\hat{b}(t), \hat{b}(t)\rangle}}{2}, \\
\Delta Y & =\frac{\sqrt{1+2\left\langle\hat{b}^{\dagger}(t), \hat{b}(t)\right\rangle-2 \operatorname{Re}\langle\hat{b}(t), \hat{b}(t)\rangle}}{2},
\end{aligned}
$$

where $\left\langle\hat{O}, \hat{O}^{\prime}\right\rangle \equiv\left\langle\hat{O} \hat{O}^{\prime}\right\rangle-\langle\hat{O}\rangle\left\langle\hat{O}^{\prime}\right\rangle$. From Eqs. (27) and (28), we can confirm that both $\left\langle\hat{b}^{\dagger}(t), \hat{b}(t)\right\rangle$ and $\langle\hat{b}(t), \hat{b}(t)\rangle$ reduce to the following time-independent quantities:

$$
\begin{gathered}
\left\langle\hat{b}^{\dagger}, \hat{b}\right\rangle=\int_{0}^{\infty} d q\left|\beta_{2}(q)\right|^{2}, \\
\langle\hat{b}, \hat{b}\rangle=-\int_{0}^{\infty} d q \beta_{1}^{*}(q) \beta_{2}(q),
\end{gathered}
$$

and that the quadrature fluctuations, $\Delta X$ and $\Delta Y$, are identical to those of the vacuum fluctuations. The integrals appearing in Eqs. (36) and (37) can be performed analytically for the Drude coupling (Appendix C). Figure 5 plots the dependencies of $\Delta X$ and $\Delta Y$ on the coupling strength $f$. We observe that there exists squeezing in $Y$ quadrature. The state is not a minimum uncertainty state, since $\sqrt{\Delta X \Delta Y}>1 / 2$.

\section{Waveguide amplitude}

From Eqs. (22) and (28), the amplitude of the waveguide field in the wave-number representation is given by

$$
\begin{aligned}
\left\langle\hat{c}_{k}(t)\right\rangle= & \sqrt{2 \pi} E_{d} \int_{0}^{\infty} d q\left[e^{-i q t} \gamma_{1}^{*}(q, k) \gamma_{1}\left(q, k_{d}\right)-e^{i q t} \gamma_{2}(q, k) \gamma_{2}^{*}\left(q, k_{d}\right)\right] \\
& +\sqrt{2 \pi} E_{d}^{*} \int_{0}^{\infty} d q\left[e^{-i q t} \gamma_{1}^{*}(q, k) \gamma_{2}\left(q, k_{d}\right)-e^{i q t} \gamma_{2}(q, k) \gamma_{1}^{*}\left(q, k_{d}\right)\right] .
\end{aligned}
$$

Using Eqs. (17)-(19), this quantity is rewritten as follows:

$$
\begin{aligned}
\left\langle\hat{c}_{k}(t)\right\rangle= & \sqrt{2 \pi} E_{d}\left[e^{-i k_{d} t} \delta\left(k-k_{d}\right)+e^{-i k t} \widetilde{\gamma}_{1}\left(k, k_{d}\right)+e^{-i k_{d} t} \tilde{\gamma}_{1}^{*}\left(k_{d}, k\right)\right] \\
& -i \sqrt{2 / \pi} \omega_{1} \xi_{k} \xi_{k_{d}} E_{d} \int_{-\infty}^{\infty} d q \frac{e^{-i q t}}{(q-k+i 0)\left(q-k_{d}-i 0\right)}\left(\frac{1}{q^{2}-\omega_{1}^{2} z(q)}-\frac{1}{q^{2}-\omega_{1}^{2} z^{*}(q)}\right) \\
& +\sqrt{2 \pi} E_{d}^{*}\left[e^{-i k t} \gamma_{2}\left(k, k_{d}\right)-e^{i k_{d} t} \gamma_{2}\left(k_{d}, k\right)\right] \\
& +i \sqrt{2 / \pi} \omega_{1} \xi_{k} \xi_{k_{d}} E_{d}^{*} \int_{-\infty}^{\infty} d q \frac{e^{i q t}}{(q+k-i 0)\left(q-k_{d}+i 0\right)}\left(\frac{1}{q^{2}-\omega_{1}^{2} z(q)}-\frac{1}{q^{2}-\omega_{1}^{2} z^{*}(q)}\right) .
\end{aligned}
$$

The integral in the second line in the above equation can be performed by employing the residue theorem. The integrand has four poles in the lower complex plane of $q$ at $k-i 0, \lambda_{1}^{*}, \lambda_{2}^{*}$, and $\lambda_{3}^{*}$, and the latter three poles yield transient components. Therefore, the stationary component of the second line comes from the pole at $k-i 0$ and is given by $-\sqrt{8 \pi} \omega_{1} \xi_{k} \xi_{k_{d}} \frac{E_{d} e^{-i k t}}{k-k_{d}-i 0}\left(\frac{1}{k^{2}-\omega_{1}^{2} z(k)}-\frac{1}{k^{2}-\omega_{1}^{2} z^{*}(k)}\right)$. Repeating the same arguments, the stationary component of the fourth line of Eq. (39) is given by $-\sqrt{8 \pi} \omega_{1} \xi_{k} \xi_{k_{d}} \frac{E_{d}^{*} e^{-i k t}}{k+k_{d}}\left(\frac{1}{k^{2}-\omega_{1}^{2} z(k)}-\right.$ $\left.\frac{1}{k^{2}-\omega_{1}^{2} z^{*}(k)}\right)$. As a result, the stationary component of the waveguide amplitude is written as

$$
\begin{gathered}
\left\langle c_{k}(t)\right\rangle=\left\langle c_{k}(t)\right\rangle^{(1)}+\left\langle c_{k}(t)\right\rangle^{(2)}+\left\langle c_{k}(t)\right\rangle^{(3)}, \\
\left\langle c_{k}(t)\right\rangle^{(1)}=\sqrt{2 \pi} \delta\left(k-k_{d}\right) E_{d} e^{-i k_{d} t},
\end{gathered}
$$




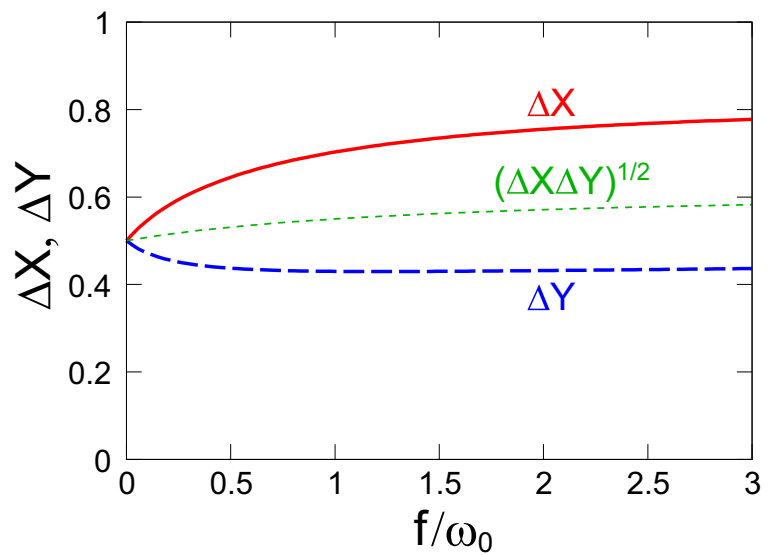

FIG. 5. Quadrature fluctuations: $\Delta X$ (solid), $\Delta Y$ (dashed), and $\sqrt{\Delta X \Delta Y}$ (thin dotted). $\omega_{x} / \omega_{0}=5$.

$$
\begin{aligned}
& \left\langle c_{k}(t)\right\rangle^{(2)}=\frac{\sqrt{8 \pi} \omega_{1} \xi_{k} \xi_{k_{d}} E_{d}}{k-k_{d}-i 0}\left(\frac{e^{-i k t}}{k^{2}-\omega_{1}^{2} z^{*}(k)}-\frac{e^{-i k_{d} t}}{k_{d}^{2}-\omega_{1}^{2} z^{*}\left(k_{d}\right)}\right), \\
& \left\langle c_{k}(t)\right\rangle^{(3)}=\frac{\sqrt{8 \pi} \omega_{1} \xi_{k} \xi_{k_{d}} E_{d}^{*}}{k+k_{d}}\left(\frac{e^{-i k t}}{k^{2}-\omega_{1}^{2} z^{*}(k)}-\frac{e^{i k_{d} t}}{k_{d}^{2}-\omega_{1}^{2} z\left(k_{d}\right)}\right) .
\end{aligned}
$$

We switch to the real-space representation $\left\langle\widetilde{c}_{r}(t)\right\rangle$, using Eq. (11). $\left\langle\widetilde{c}_{r}(t)\right\rangle^{(1)}$ is immediately given by

$$
\left\langle\widetilde{c}_{r}(t)\right\rangle^{(1)}=E_{d} e^{i k_{d}(r-t)}=E(r, t) .
$$

Obviously, this is nothing but the input drive field [Eq. (9)]. Regarding $\left\langle\widetilde{c}_{r}(t)\right\rangle^{(2)}$, the principal contribution comes from the pole at $k=k_{d}+i 0$ in the righthand side of Eq. (42). Therefore, we can employ the following approximation, $\left\langle c_{k}(t)\right\rangle_{s}^{(2)} \approx \sqrt{8 \pi} \omega_{1} \xi_{k_{d}}^{2} E_{d}\left[k_{d}^{2}-\right.$ $\left.\omega_{1}^{2} z^{*}\left(k_{d}\right)\right]^{-1}\left[k-k_{d}-i 0\right]^{-1}\left(e^{-i k t}-e^{-i k_{d} t}\right)$. Then, we have

$$
\left\langle\widetilde{c}_{r}(t)\right\rangle^{(2)} \approx-\frac{4 \pi i \omega_{1} \xi_{k_{d}}^{2}}{k_{d}^{2}-\omega_{1}^{2} z^{*}\left(k_{d}\right)} \theta(r) \theta(t-r) E(r, t),
$$

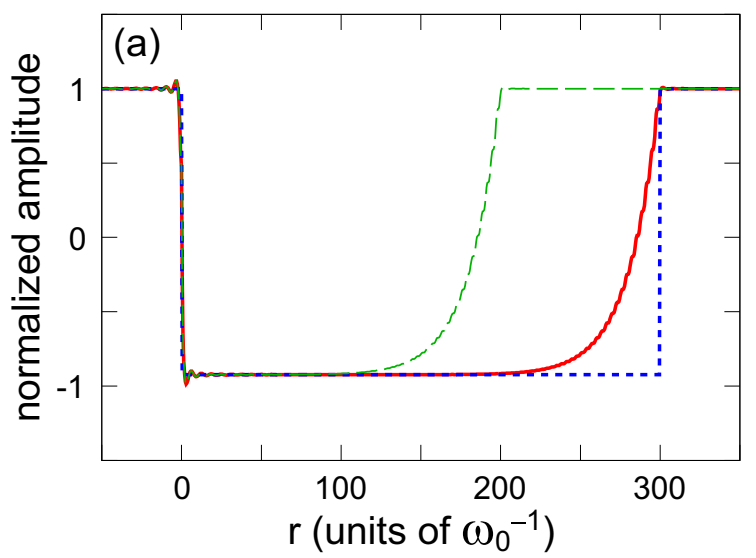

where $\theta$ is the Heaviside step function. This represents the radiation from the oscillator emitted into the positive $r$ region. In contrast, regarding $\left\langle\widetilde{c}_{r}(t)\right\rangle^{(3)}$, the pole at $k=-k_{d}$ in the right-hand side of Eq. (43) does not yield a significant contribution since the integration range in Eq. (11) is restricted to the positive $k$ region. Combining these results, we obtain the following analytic form of $\left\langle\widetilde{c}_{r}(t)\right\rangle$ :

$$
\left\langle\widetilde{c}_{r}(t)\right\rangle \approx\left(1-\frac{4 \pi i \omega_{1} \xi_{k_{d}}^{2}}{k_{d}^{2}-\omega_{1}^{2} z^{*}\left(k_{d}\right)} \theta(r) \theta(t-r)\right) \times E(r, t) .
$$

The spatial shape of the normalized amplitude, $\left\langle\widetilde{c}_{r}(t)\right\rangle / E(r, t)$, is plotted in Fig. 6. If the oscillator is uncoupled to the waveguide, the drive field propagates freely in the waveguide, namely, $\left\langle\widetilde{c}_{r}(t)\right\rangle=E(r, t)$, and therefore the real (imaginary) part of the normalized amplitude is unity (zero) at any $r$. Such free propagation is observed in Fig. 6 in the $r<0$ and $t<r$ regions. In contrast, when the oscillator is coupled to the waveguide, radiation from the driven oscillator is overlapped onto the freely propagating field in the $0<r<t$ region. Under the parameters of Fig. 6, the reflection coefficient [Eq. (47)] amounts to $R=(-12+5 i) / 13$. The normalized amplitude at the output port is $\operatorname{Re}(R)=-0.923$ and $\operatorname{Im}(R)=0.385$, as we observe in Fig. 6.

The rigorous shape of $\left\langle\widetilde{c}_{r}(t)\right\rangle$ [numerical Fourier transform of Eq. (40)] and the approximate one [Eq. (46)] are compared in Fig. 6. We observe good agreement between them, except deviations at the wavefront of radiation $(r \sim t)$ and at the oscillator position $(r \sim 0)$. The former deviation originates in the transient oscillator response, which is neglected when deriving Eq. (46). The transient response vanishes within a timescale of $\widetilde{\kappa}^{-1}$, which agrees with our observation in Fig. 6. On the other hand, the latter deviation around the oscillator position originates in the fact that the oscillator-waveguide coupling has a finite bandwidth and therefore is not spatially local. The bandwidth is of the order of $\omega_{0}$ in the wave-number space due to the lower cutoff at $k=0$, and is therefore of the order of $\omega_{0}^{-1}$ in the real space. This explains the deviation around the origin in Fig. 6.

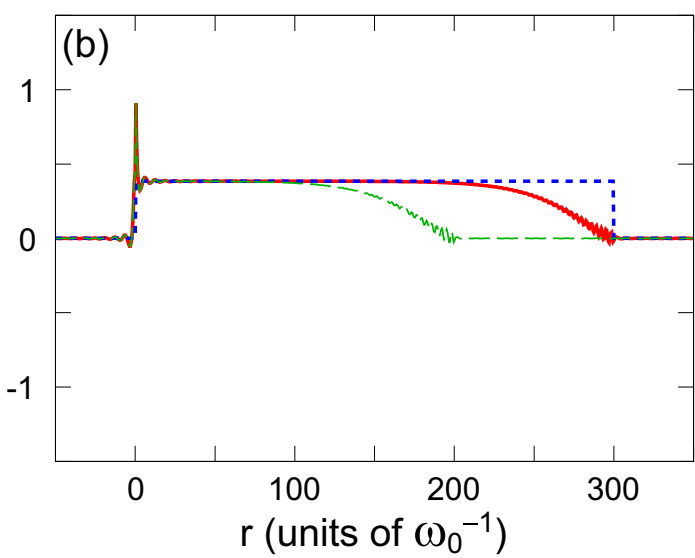

FIG. 6. Normalized amplitude of the waveguide field, $\left\langle\widetilde{c}_{r}(t)\right\rangle / E(r, t)$. (a) Real and (b) imaginary parts. Solid (thin dashed) lines represent the rigorous amplitudes at $t=300 / \omega_{0}\left(200 / \omega_{0}\right)$ and dotted lines represent the approximate ones [Eq. (46)] at $t=300 / \omega_{0}$. The other parameters are $f / \omega_{0}=0.1\left(\widetilde{\kappa} / \omega_{0}=0.0978\right)$ and $k_{d} / \omega_{0}=1$. 


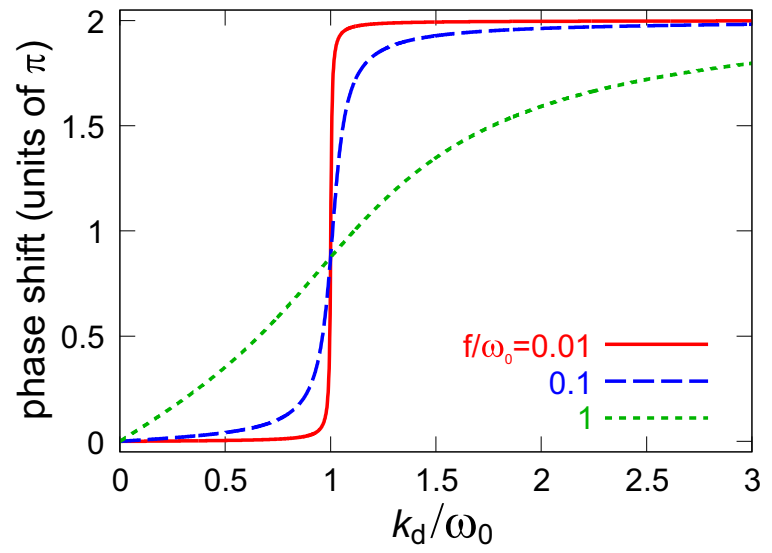

FIG. 7. Phase shift upon reflection as a function of the drive frequency. The oscillator-waveguide coupling strength $f$ is indicated.

A notable fact is that, in contrast with the oscillator amplitude [Eq. (30)] that is composed of both positively and negatively oscillating components, the waveguide field amplitude in the output port [Eq. (46)] is composed only of the positively oscillating one $[42,43]$. Therefore, the elliptic motion is specific to the oscillator amplitude.

\section{Reflection coefficient}

The reflection coefficient is identified as $R=$ $\left\langle\widetilde{c}_{r}(t)\right\rangle / E(r, t)$ at the output port $(r>0)$. From Eq. (46), $R$ is given by

$$
R\left(k_{d}\right)=1-\frac{4 \pi i \omega_{1} \xi_{k_{d}}^{2}}{k_{d}^{2}-\omega_{1}^{2} z^{*}\left(k_{d}\right)} .
$$

We can check that $|R|=1$ for any drive frequency $k_{d}$. This implies that input field is reflected completely coherently, which is characteristic to linear optical response. In Fig. 7, we plot the phase shift upon reflection, $\arg R$, as a function of $k_{d}$, varying the coupling strength $f$. We observe the broadening of the linewidth as we increase the coupling. The spectrum takes a kink-shaped form around the resonance. For a weak coupling, the spectrum is antisymmetric with respect to the resonance frequency, in accordance with the standard input-output theory. However, for a stronger coupling, such symmetry is gradually lost.

We define the dressed resonance frequency $\widetilde{\omega}$ as the drive frequency achieving the $\pi$ phase shift, $R(\widetilde{\omega})=-1$. From this equation, $\widetilde{\omega}$ is analytically given by

$$
\widetilde{\omega}^{2}=\frac{\sqrt{\left(\omega_{x}^{2}-\omega_{0}^{2}-f \omega_{x}\right)^{2}+4 \omega_{0}^{2} \omega_{x}^{2}}-\left(\omega_{x}^{2}-\omega_{0}^{2}-f \omega_{x}\right)}{2} .
$$

We observe in Fig. 7 that the reflection coefficient becomes independent of the coupling strength at the bare cavity resonance, $k_{d}=\omega_{0}$; we can check that $R\left(\omega_{0}\right)=\left(i \omega_{0}-\right.$ $\left.\omega_{x}\right) /\left(i \omega_{0}+\omega_{x}\right)$.

\section{E. Open waveguide}

In Sec. IV D, we evaluated the reflection coefficient $R$ when a semi-infinite waveguide is coupled to the oscillator. From this result, we can readily determine the reflection and transmission coefficients $R^{\prime}$ and $T^{\prime}$, when the oscillator is coupled to an open waveguide [Fig. 8(a)]. The amplitude of the waveguide field in this case is written as

$$
E(r, t)=E_{d} e^{-i \omega_{d} t} \times \begin{cases}e^{i k_{d} r}+R^{\prime} e^{-i k_{d} r} & (r<0) \\ T^{\prime} e^{i k_{d} r} & (0<r) .\end{cases}
$$

We divide this field into even and odd components. The even component interacts with the oscillator, whereas the odd component does not. The even component is defined by $E_{s}(r, t)=[E(r, t)+E(-r, t)] / 2$ and is therefore given by $E_{s}(r, t)=\frac{1}{2} E_{d} e^{-i k_{d}(r+t)}+\frac{R^{\prime}+T^{\prime}}{2} E_{d} e^{i k_{d}(r-t)}$ for $r>0$. Since the first (second) term in the right-hand side of this equation represents the incoming (outgoing) field, we have $R^{\prime}+T^{\prime}=$ $R$. Similarly, the odd component is defined by $E_{a}(r, t)=$ $[E(r, t)-E(-r, t)] / 2$ and is therefore given by $E_{s}(r, t)=$ $-\frac{1}{2} E_{d} e^{-i k_{d}(r+t)}+\frac{T^{\prime}-R^{\prime}}{2} E_{d} e^{i k_{d}(r-t)}$ for $r>0$. Since the incoming field simply transmits the oscillator without interaction, we have $T^{\prime}-R^{\prime}=1$. Therefore,

$$
\begin{aligned}
& R^{\prime}=(R-1) / 2, \\
& T^{\prime}=(R+1) / 2 .
\end{aligned}
$$

We can readily confirm that $\left|R^{\prime}\right|^{2}+\left|T^{\prime}\right|^{2}=1$. The transmissivity $\left|T^{\prime}\right|^{2}$ is plotted in Fig. 8(b) as a function of the drive
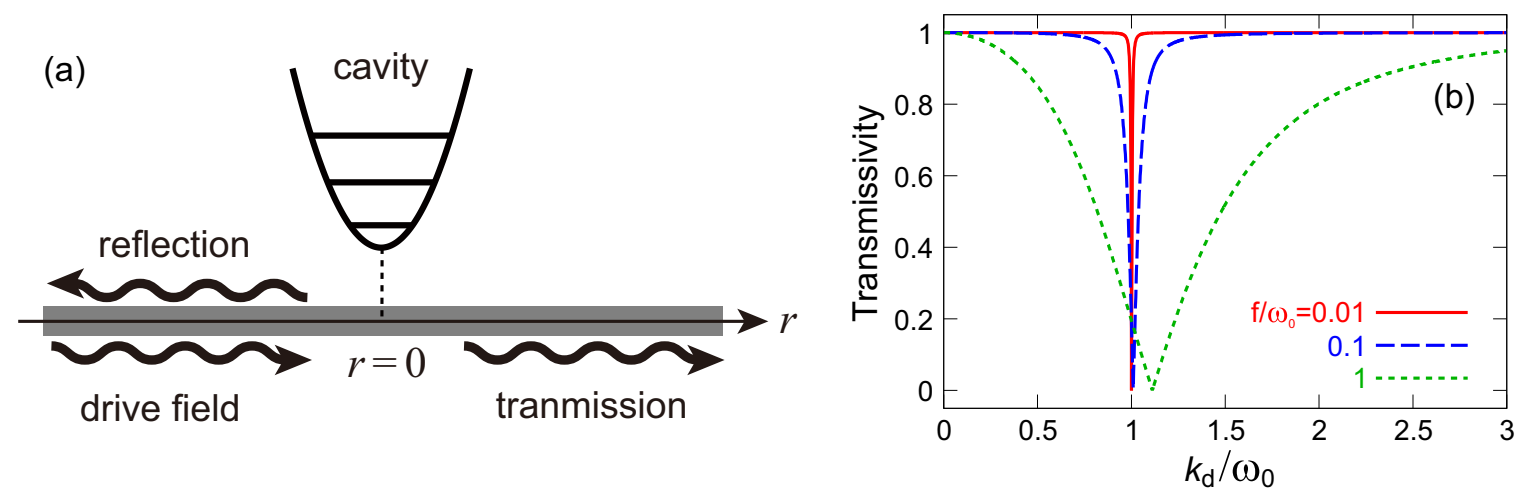

FIG. 8. (a) Schematic of an oscillator coupled to an open waveguide. (b) Transmissivity $\left|T^{\prime}\right|^{2}$ as a function of the drive frequency. The oscillator-waveguide coupling strength $f$ is indicated. 

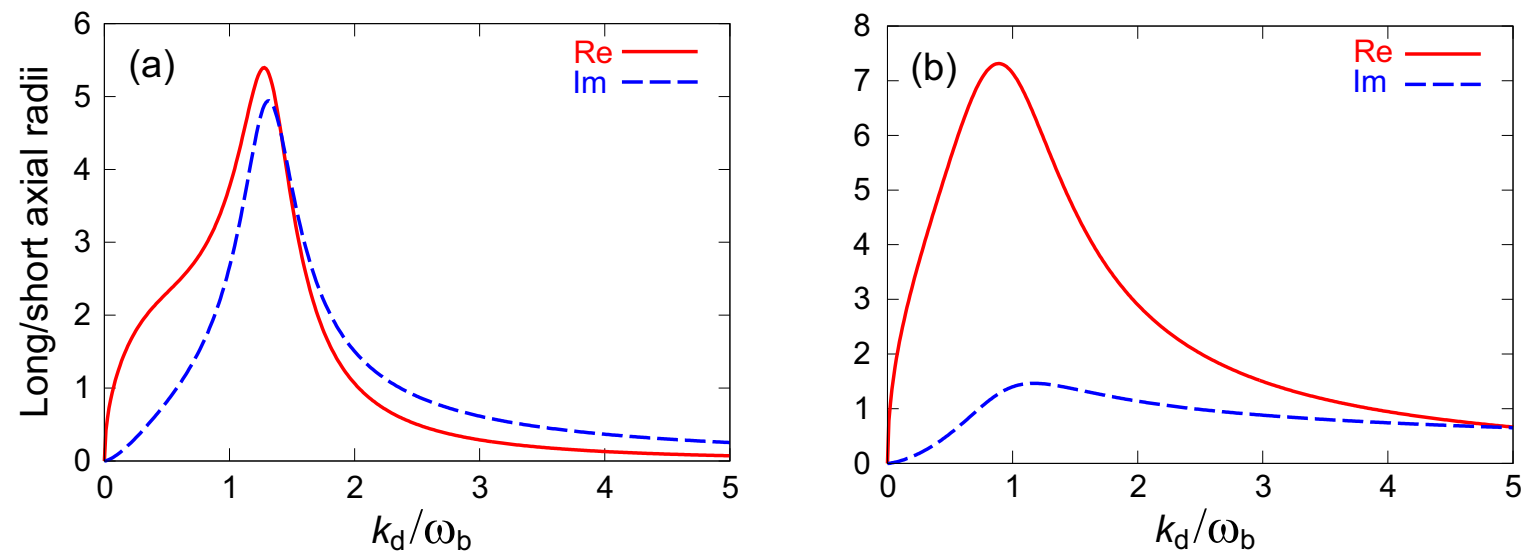

FIG. 9. The same plot as Fig. 4(d) for different cutoff frequency $\omega_{x}$. (a) $\omega_{x} / \omega_{0}=1$ and $f / \omega_{0}=1$. (b) $\omega_{x} / \omega_{0}=25$ and $f / \omega_{0}=1$.

frequency. We observe that the symmetric transmission dip for a weak-coupling case (solid line) gradually becomes asymmetric as the oscillator-waveguide coupling increases (dashed and dotted lines).

\section{SUMMARY}

We investigated optical response of a linear waveguide QED system, namely, a bosonic oscillator coupled to a waveguide. Our analysis is based on exact diagonalization of the overall Hamiltonian, and is therefore rigorous even in the ultra- and deep-strong-coupling regimes of waveguide QED. Owing to the counter-rotating terms in the oscillatorwaveguide coupling, the phase-space motion of the oscillator amplitude is elliptical in general, and this becomes remarkable in the ultrastrong coupling regime. However, such an elliptical motion does not appear in the output field, contrary to the intuition by the standard input-output theory. We obtained an analytic expression of the reflection/transmission coefficient, which becomes asymmetric with respect to the resonance frequency for stronger coupling. Although the present work is based on a specific Drude coupling, the features observed here would apply generally in ultrastrong waveguide QED.

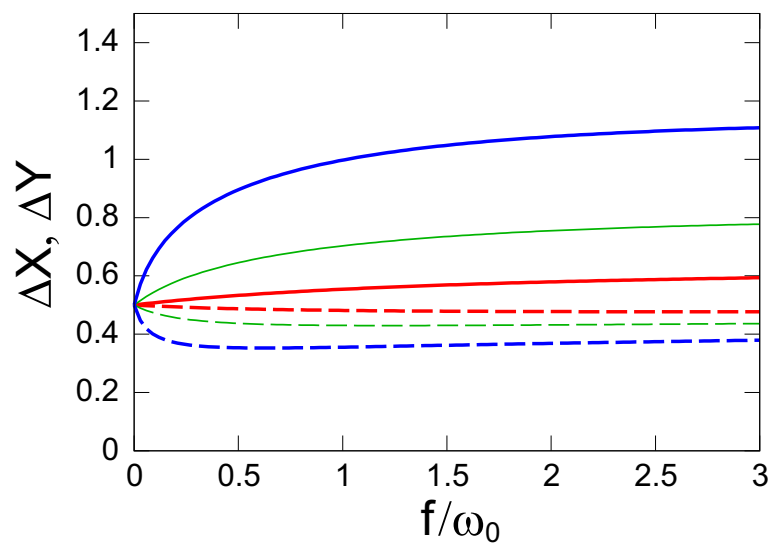

FIG. 10. Quadrature fluctuations $\Delta X$ (solid) and $\Delta Y$ (dashed) for different cutoff frequency $\omega_{x}$. Red, thin green, and blue lines are the results for $\omega_{x} / \omega_{0}=1,5$, and 25 , respectively.

\section{ACKNOWLEDGMENTS}

The author acknowledges fruitful discussions with T. Shitara and I. Iakoupov. This work is supported in part by JST CREST (Grant No. JPMJCR1775), JST ERATO (Grant No. JPMJER1601), MEXT Q-LEAP, and JSPS KAKENHI (Grant No. 19K03684).

\section{APPENDIX A: DETERMINATION OF $\beta_{1,2}$ AND $\gamma_{1,2}$}

From Eqs. (12) and (13), we have $\left[\hat{d}_{k}, \hat{H}\right]=k \hat{d}_{k}$. This leads to the following equations:

$$
\begin{gathered}
\left(k-\omega_{1}\right) \beta_{1}(k)=\int_{0}^{\infty} d q \xi_{q}\left[\gamma_{1}(k, q)-\gamma_{2}(k, q)\right], \\
\left(k+\omega_{1}\right) \beta_{2}(k)=\int_{0}^{\infty} d q \xi_{q}\left[\gamma_{1}(k, q)-\gamma_{2}(k, q)\right], \\
(k-q) \gamma_{1}(k, q)=\xi_{q}\left[\beta_{1}(k)-\beta_{2}(k)\right], \\
(k+q) \gamma_{2}(k, q)=\xi_{q}\left[\beta_{1}(k)-\beta_{2}(k)\right] .
\end{gathered}
$$

From Eqs. (A2) and (A4), we obtain $\beta_{2}(k)=\frac{k-\omega_{1}}{k+\omega_{1}} \beta_{1}(k)$ and $\gamma_{2}(k, q)=\frac{k-q}{k+q} \gamma_{1}(k, q)$. Then, Eqs. (A1) and (A3) are rewritten as

$$
\begin{gathered}
\left(k-\omega_{1}\right) \beta_{1}(k)=2 \int_{0}^{\infty} d q \frac{q \xi_{q}}{k+q} \gamma_{1}(k, q), \\
(k-q) \gamma_{1}(k, q)=\frac{2 \omega_{1}}{k+\omega_{1}} \beta_{1}(k) \xi_{q} .
\end{gathered}
$$

Equation (A6) is rewritten as

$$
\gamma_{1}(k, q)=\frac{2 \omega_{1}}{k+\omega_{1}} \beta_{1}(k) \xi_{q}\left(\frac{1}{k-q-i 0}+y(k) \delta(k-q)\right),
$$

where $y(k)$ is a quantity to be determined. Substituting the above equation into Eq. (A5), and using $\int_{0}^{\infty} \frac{q \xi_{q}^{2}}{(k+q)(k-q-i 0)}=$ $\frac{1}{2} \int_{-\infty}^{\infty} \frac{\xi_{q}^{2}}{k-q-i 0}, y(k)$ is given by

$$
\begin{gathered}
y(k)=\frac{1}{\xi_{k}^{2}}\left(\frac{k^{2}-\omega_{1}^{2}}{2 \omega_{1}}-\Sigma(k)\right), \\
\Sigma(k)=\int_{-\infty}^{\infty} d q \frac{\xi_{q}^{2}}{k-q-i 0} .
\end{gathered}
$$



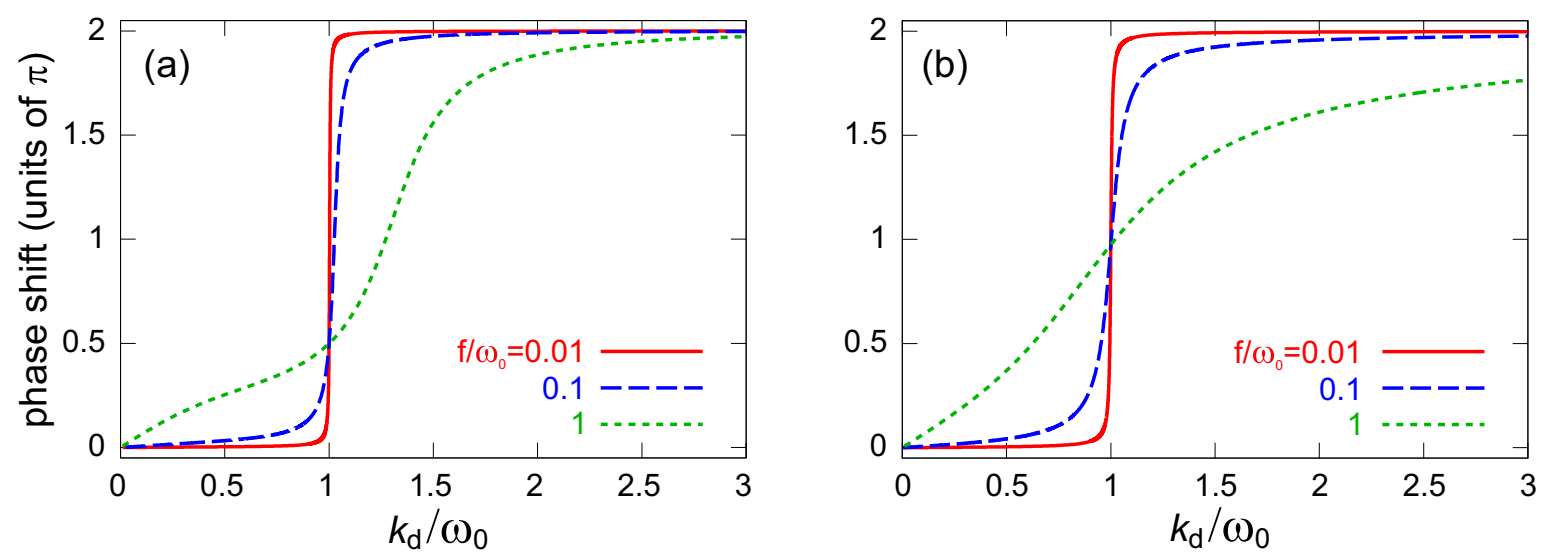

FIG. 11. The same plot as Fig. 7 for different cutoff frequency $\omega_{x}$. (a) $\omega_{x} / \omega_{0}=1$. (b) $\omega_{x} / \omega_{0}=25$.

Note that $\Sigma(k)$ is the self-energy of the oscillator, satisfying $\Sigma(-k)=\Sigma^{*}(k)$ and $\operatorname{Im} \Sigma(k)=\pi \xi_{k}^{2}$.

Up to here, we derived the expressions of $\beta_{2}, \gamma_{1}$, and $\gamma_{2}$ in terms of $\beta_{1} . \beta_{1}(k)$ is determined by the normalization condition, Eq. (13). This is rewritten as $\delta\left(k-k^{\prime}\right)=\beta_{1}(k) \beta_{1}^{*}\left(k^{\prime}\right)-$ $\beta_{2}(k) \beta_{2}^{*}\left(k^{\prime}\right)+\int_{0}^{\infty} d q\left[\gamma_{1}(k, q) \gamma_{1}^{*}\left(k^{\prime}, q\right)-\gamma_{2}(k, q) \gamma_{2}^{*}\left(k^{\prime}, q\right)\right]$, which leads to $\frac{2 \omega_{1} \xi_{k}}{\left(k+\omega_{1}\right)}\left|\beta_{1}(k)\right||y(k)|=1$. By adequately choosing the phase of $\beta_{1}$, we obtain Eq. (15),

$$
\beta_{1}(k)=\frac{k+\omega_{1}}{2 \omega_{1} \xi_{k} y(k)}=\frac{\left(k+\omega_{1}\right) \xi_{k}}{k^{2}-\omega_{1}^{2} z(k)} .
$$

$\beta_{2}, \gamma_{1}$, and $\gamma_{2}$ are obtained accordingly.

\section{APPENDIX B: TRANSIENT COMPONENT OF OSCILLATOR AMPLITUDE}

Here we present the transient component of the oscillator amplitude $\langle\hat{b}(t)\rangle_{t}$, which is omitted in Sec. IV A:

$$
\begin{aligned}
\langle\hat{b}(t)\rangle_{t}= & \sqrt{8 \pi} E_{d} \omega_{1} \xi_{k_{d}} \int_{-\infty}^{\infty} d q \frac{e^{-i q t}\left(q+\omega_{1}\right) \xi_{q}^{2}}{\left(q-k_{d}-i 0\right)\left[q^{2}-\omega_{1}^{2} z(q)\right]\left[q^{2}-\omega_{1}^{2} z^{*}(q)\right]} \\
& -\sqrt{8 \pi} E_{d}^{*} \omega_{1} \xi_{k_{d}} \int_{-\infty}^{\infty} d q \frac{e^{i q t}\left(q-\omega_{1}\right) \xi_{q}^{2}}{\left(q-k_{d}+i 0\right)\left[q^{2}-\omega_{1}^{2} z(q)\right]\left[q^{2}-\omega_{1}^{2} z^{*}(q)\right]}
\end{aligned}
$$

Using $\frac{\omega_{1} \xi_{q}^{2}}{\left[q^{2}-\omega_{1}^{2} z(q)\right]\left[q^{2}-\omega_{1}^{2} z^{*}(q)\right]}=\frac{1}{4 i \pi}\left(\frac{1}{q^{2}-\omega_{1}^{2} z(q)}-\frac{1}{q^{2}-\omega_{1}^{2} z^{*}(q)}\right)$ and that $\frac{1}{q^{2}-\omega_{1}^{2} z(q)}$ has no poles on the lower half plane, the transient component is rewritten as

$$
\langle b(t)\rangle_{t}=\frac{i E_{d} \xi_{k_{d}}}{\sqrt{2 \pi}} \int_{-\infty}^{\infty} d q \frac{e^{-i q t}\left(q+\omega_{1}\right)}{\left(q-k_{d}-i 0\right)\left[q^{2}-\omega_{1}^{2} z^{*}(q)\right]}+\frac{i E_{d}^{*} \xi_{k_{d}}}{\sqrt{2 \pi}} \int_{-\infty}^{\infty} d q \frac{e^{i q t}\left(q-\omega_{1}\right)}{\left(q-k_{d}+i 0\right)\left[q^{2}-\omega_{1}^{2} z(q)\right]}
$$

\section{APPENDIX C: INTEGRALS IN EQS. (36) AND (37)}

Here, we derive an analytical form of the integral in the right-hand side of Eq. (36). From Eq. (20), we have $z(k)-z^{*}(k)=$ $4 i \pi \xi_{k}^{2} / \omega_{1}$. Therefore, the integral is rewritten as

$$
\int_{0}^{\infty} d q\left|\beta_{2}(q)\right|^{2}=\frac{1}{4 i \pi \omega_{1}}\left(\int_{0}^{\infty} d q \frac{\left(q-\omega_{1}\right)^{2}}{q^{2}-\omega_{1}^{2} z(q)}-\text { c.c. }\right) .
$$

We denote the integrand in the right-hand side of Eq. (C1) by $f(q)$. Using Eq. (24), $f(q)$ is rewritten as

$$
f(q)=\frac{\left(q-\omega_{1}\right)^{2}}{q^{2}-\omega_{1}^{2} z(q)}=\frac{\left(q-\omega_{1}\right)^{2}\left(q-i \omega_{x}\right)}{\left(q-\lambda_{1}\right)\left(q-\lambda_{2}\right)\left(q-\lambda_{3}\right)}=1+\sum_{j=1}^{3} \frac{c_{j}}{q-\lambda_{j}},
$$

where $c_{j}$ is a residue of $f(q)$ at $q=\lambda_{j}$. Substituting Eq. (C2) into Eq. (C1), we obtain

$$
\int_{0}^{\infty} d q\left|\beta_{2}(q)\right|^{2}=-\frac{1}{2 \pi \omega_{1}} \sum_{j=1}^{3} \operatorname{Im}\left\{c_{j} \ln \left(-\lambda_{j}\right)\right\} .
$$


Repeating the same argument, the integral appearing in Eq. (37) is given by

$$
-\int_{0}^{\infty} d q \beta_{1}^{*}(q) \beta_{2}(q)=-\frac{1}{2 \pi \omega_{1}} \sum_{j=1}^{3} \operatorname{Im}\left\{d_{j} \ln \left(-\lambda_{j}\right)\right\},
$$

where $d_{j}$ is a residue at $q=\lambda_{j}$ of the following function $g(q)$,

$$
g(q)=\frac{\left(\omega_{1}^{2}-q^{2}\right)\left(q-i \omega_{x}\right)}{\left(q-\lambda_{1}\right)\left(q-\lambda_{2}\right)\left(q-\lambda_{3}\right)} .
$$

\section{APPENDIX D: DERIVATION OF EQ. (39)}

Here, we present the derivation of Eq. (39) from Eq. (38). In particular, we focus on the first integral on the right-hand side of Eq. (38), $I=\sqrt{2 \pi} E_{d} \int_{0}^{\infty} d q\left[e^{-i q t} \gamma_{1}^{*}(q, k) \gamma_{1}\left(q, k_{d}\right)-e^{i q t} \gamma_{2}(q, k) \gamma_{2}^{*}\left(q, k_{d}\right)\right]$. Using Eq. (17), this quantity is rewritten as $I=I_{1}+I_{2}+I_{3}$, where

$$
\begin{gathered}
I_{1}=\sqrt{2 \pi} E_{d}\left[e^{-i k_{d} t} \delta\left(k-k_{d}\right)+e^{-i k t} \tilde{\gamma}_{1}\left(k, k_{d}\right)+e^{-i k_{d} t} \widetilde{\gamma}_{1}^{*}\left(k_{d}, k\right)\right], \\
I_{2}=\sqrt{2 \pi} E_{d} \int_{0}^{\infty} d q e^{-i q t} \widetilde{\gamma}_{1}^{*}(q, k) \widetilde{\gamma}_{1}\left(q, k_{d}\right), \\
I_{3}=-\sqrt{2 \pi} E_{d} \int_{0}^{\infty} d q e^{i q t} \gamma_{2}(q, k) \gamma_{2}^{*}\left(q, k_{d}\right) .
\end{gathered}
$$

$I_{1}$ is the first line of the right-hand side of Eq. (39). Using Eq. (19), $I_{2}$ is rewritten as

$$
I_{2}=4 \sqrt{2 \pi} \omega_{1}^{2} \xi_{k} \xi_{k_{d}} E_{d} \int_{0}^{\infty} d q \frac{e^{-i q t} \xi_{q}^{2}}{(q-k+i 0)\left(q-k_{d}-i 0\right)\left[q^{2}-\omega_{1}^{2} z(q)\right]\left[q^{2}-\omega_{1}^{2} z^{*}(q)\right]} .
$$

From Eq. (20), we obtain $z(q)-z^{*}(q)=4 i \pi \xi_{q}^{2} / \omega_{1}$. Therefore,

$$
\frac{\xi_{q}^{2}}{\left[q^{2}-\omega_{1}^{2} z(q)\right]\left[q^{2}-\omega_{1}^{2} z^{*}(q)\right]}=\frac{1}{4 i \pi \omega_{1}}\left(\frac{1}{q^{2}-\omega_{1}^{2} z(q)}-\frac{1}{q^{2}-\omega_{1}^{2} z^{*}(q)}\right) .
$$

Substituting Eq. (D5) into Eq. (D4), we have

$$
I_{2}=-i \sqrt{2 / \pi} \omega_{1} \xi_{k} \xi_{k_{d}} E_{d} \int_{0}^{\infty} d q \frac{e^{-i q t}}{(q-k+i 0)\left(q-k_{d}-i 0\right)}\left(\frac{1}{q^{2}-\omega_{1}^{2} z(q)}-\frac{1}{q^{2}-\omega_{1}^{2} z^{*}(q)}\right) .
$$

Repeating the same arguments, $I_{3}$ is rewritten as

$$
I_{3}=i \sqrt{2 / \pi} \omega_{1} \xi_{k} \xi_{k_{d}} E_{d} \int_{0}^{\infty} d q \frac{e^{i q t}}{(q+k)\left(q+k_{d}\right)}\left(\frac{1}{q^{2}-\omega_{1}^{2} z(q)}-\frac{1}{q^{2}-\omega_{1}^{2} z^{*}(q)}\right) .
$$

By switching the integral variable $(q \rightarrow-q)$ in Eq. (D7) and using $z^{*}(q)=z(-q)$, we finally have

$$
I_{2}+I_{3}=-i \sqrt{2 / \pi} \omega_{1} \xi_{k} \xi_{k_{d}} E_{d} \int_{-\infty}^{\infty} d q \frac{e^{-i q t}}{(q-k+i 0)\left(q-k_{d}-i 0\right)}\left(\frac{1}{q^{2}-\omega_{1}^{2} z(q)}-\frac{1}{q^{2}-\omega_{1}^{2} z^{*}(q)}\right),
$$

which is the second line of the right-hand side of Eq. (39).

\section{APPENDIX E: EFFECTS OF CUTOFF FREQUENCY}

Throughout this study, we fixed the cutoff frequency of the Drude coupling at $\omega_{x}=5 \omega_{0}$. Here, we present the numerical results for smaller $\left(\omega_{x}=\omega_{0}\right)$ and larger $\left(\omega_{x}=25 \omega_{0}\right)$ cutoff frequencies. Figure 9 plots the long/short axial radii of the phasespace rotation, and Fig. 10 plots the quadrature fluctuations. We observe that elliptic phase-space motion and the quadrature squeezing are more stressed for larger cutoff frequency. This is attributed to the fact the net oscillator-waveguide coupling, $\int_{0}^{\infty} d k \eta_{k}^{2}=f \omega_{x}$, is larger for higher $\omega_{x}$. The reflection coefficient plotted in Fig. 11 is not affected by $\omega_{x}$ significantly. These observations indicate that the cutoff frequency (more generally, the functional form of the coupling) affects the results of the main part of this paper quantitatively, but retains their qualitative features.

[1] M. Brune, F. Schmidt-Kaler, A. Maali, J. Dreyer, E. Hagley, J. M. Raimond, and S. Haroche, Quantum Rabi Oscillation: A
Direct Test of Field Quantization in a Cavity, Phys. Rev. Lett. 76, 1800 (1996). 
[2] J. McKeever, A. Boca, A. D. Boozer, J. R. Buck, and H. J. Kimble, Experimental realization of a one-atom laser in the regime of strong coupling, Nature (London) 425, 268 (2003).

[3] M. Keller, B. Lange, K. Hayasaka, W. Lange, and H. Walther, Deterministic coupling of single ions to an optical cavity, Appl. Phys. B 76, 125 (2003).

[4] T. Yoshie, A. Scherer, J. Hendrickson, G. Khitrova, H. M. Gibbs, G. Rupper, C. Ell, O. B. Shchekin, and D. G. Deppe, Vacuum Rabi splitting with a single quantum dot in a photonic crystal nanocavity, Nature (London) 432, 200 (2004).

[5] G. Gunter, A. A. Anappara, J. Hees, A. Sell, G. Biasiol, L. Sorba, S. D. Liberato, C. Ciuti, A. Tredicucci, A. Leitenstorfer, and R. Huber, Sub-cycle switch-on of ultrastrong light-matter interaction, Nature (London) 458, 178 (2009).

[6] T. Niemczyk, F. Deppe, H. Huebl, E. P. Menzel, F. Hocke, M. J. Schwarz, J. J. Garcia-Ripoll, D. Zueco, T. Hummer, E. Solano, A. Marx, and R. Gross, Circuit quantum electrodynamics in the ultrastrong-coupling regime, Nat. Phys. 6, 772 (2010).

[7] S. Gambino, M. Mazzeo, A. Genco, O. D. Stefano, S. Savasta, S. Patane, D. Ballarini, F. Mangione, G. Lerario, D. Sanvitto, and G. Gigli, Exploring light-matter interaction phenomena under ultrastrong coupling regime, ACS Photonics 1, 1042 (2014).

[8] A. Bayer, M. Pozimski, S. Schambeck, D. Schuh, R. Huber, D. Bougeard, and C. Lange, Terahertz light-matter interaction beyond unity coupling strength, Nano Lett. 17, 6340 (2017).

[9] X. Li, M. Bamba, Q. Zhang, S. Fallahi, G. C. Gardner, W. Gao, M. Lou, K. Yoshioka, M. J. Manfra, and J. Kono, Vacuum Bloch-Siegert shift in Landau polaritons with ultrahigh cooperativity, Nat. Photonics 12, 324 (2018).

[10] F. Yoshihara, T. Fuse, S. Ashhab, K. Kakuyanagi, S. Saito, and K. Semba, Superconducting qubit-oscillator circuit beyond the ultrastrong-coupling regime, Nat. Phys. 13, 44 (2017).

[11] F. Yoshihara, T. Fuse, Z. Ao, S. Ashhab, K. Kakuyanagi, S. Saito, T. Aoki, K. Koshino, and K. Semba, Inversion of Qubit Energy Levels in Qubit-Oscillator Circuits in the Deep-StrongCoupling Regime, Phys. Rev. Lett. 120, 183601 (2018).

[12] A. F. Kockum, A. Miranowicz, S. De Liberato, S. Savasta, and F. Nori, Ultrastrong coupling between light and matter, Nat. Rev. Phys. 1, 19 (2019).

[13] P. Forn-Diaz, J. Lisenfeld, D. Marcos, J. J. Garcia-Ripoll, E. Solano, C. J. P. M. Harmans, and J. E. Mooij, Observation of the Bloch-Siegert Shift in a Qubit-Oscillator System in the Ultrastrong Coupling Regime, Phys. Rev. Lett. 105, 237001 (2010).

[14] S.-P. Wang, G.-Q. Zhang, Y. Wang, Z. Chen, T. Li, J. S. Tsai, S.-Y. Zhu, and J. Q. You, Photon-Dressed Bloch-Siegert Shift in an Ultrastrongly Coupled Circuit Quantum Electrodynamical System, Phys. Rev. Appl. 13, 054063 (2020).

[15] C. Ciuti, G. Bastard, and I. Carusotto, Quantum vacuum properties of the intersubband cavity polariton field, Phys. Rev. B 72, 115303 (2005).

[16] V. V. Dodonov and A. V. Dodonov, QED effects in a cavity with a time-dependent thin semiconductor slab excited by laser pulses, J. Phys. B 39, S749 (2006).

[17] A. Auer and G. Burkard, Entangled photons from the polariton vacuum in a switchable optical cavity, Phys. Rev. B 85, 235140 (2012).

[18] M. Cirio, S. De Liberato, N. Lambert, and F. Nori, Ground State Electroluminescence, Phys. Rev. Lett. 116, 113601 (2016).
[19] S. De Liberato, Virtual photons in the ground state of a dissipative system, Nat. Commun. 8, 1465 (2017).

[20] C. K. Law, Vacuum Rabi oscillation induced by virtual photons in the ultrastrong-coupling regime, Phys. Rev. A 87, 045804 (2013).

[21] L. Garziano, R. Stassi, V. Macri, A. F. Kockum, S. Savasta, and F. Nori, Multiphoton quantum Rabi oscillations in ultrastrong cavity QED, Phys. Rev. A 92, 063830 (2015).

[22] A. F. Kockum, A. Miranowicz, V. Macri, S. Savasta, and F. Nori, Deterministic quantum nonlinear optics with single atoms and virtual photons, Phys. Rev. A 95, 063849 (2017).

[23] O. Astafiev, A. M. Zagoskin, A. A. Abdumalikov, Jr., Y. A. Pashkin, T. Yamamoto, K. Inomata, Y. Nakamura, and J. S. Tsai, Resonance fluorescence of a single artificial atom, Science 327, 840 (2010).

[24] I.-C. Hoi, A. F. Kockum, T. Palomaki, T. M. Stace, B. Fan, L. Tornberg, S. R. Sathyamoorthy, G. Johansson, P. Delsing, and C. M. Wilson, Giant Cross-Kerr Effect for Propagating Microwaves Induced by an Artificial Atom, Phys. Rev. Lett. 111, 053601 (2013).

[25] A. F. van Loo, A. Fedorov, K. Lalumiere, B. C. Sanders, A. Blais, and A. Wallraff, Photon-mediated interactions between distant artificial atoms, Science 342, 1494 (2013).

[26] K. Koshino, H. Terai, K. Inomata, T. Yamamoto, W. Qiu, Z. Wang, and Y. Nakamura, Observation of Three-State Dressed States in Circuit Quantum Electrodynamics, Phys. Rev. Lett. 110, 263601 (2013).

[27] A. Goban, C.-L. Hung, J. D. Hood, S.-P. Yu, J. A. Muniz, O. Painter, and H. J. Kimble, Superradiance for Atoms Trapped along a Photonic Crystal Waveguide, Phys. Rev. Lett. 115, 063601 (2015).

[28] M. Arcari, I. Sollner, A. Javadi, S. Lindskov Hansen, S. Mahmoodian, J. Liu, H. Thyrrestrup, E. H. Lee, J. D. Song, S. Stobbe, and P. Lodahl, Near-Unity Coupling Efficiency of a Quantum Emitter to a Photonic Crystal Waveguide, Phys. Rev. Lett. 113, 093603 (2014).

[29] P. Forn-Diaz, J. J. Garcia-Ripoll, B. Peropadre, J. L. Orgiazzi, M. A. Yurtalan, R. Belyansky, C. M. Wilson, and A. Lupascu, Ultrastrong coupling of a single artificial atom to an electromagnetic continuum in the nonperturbative regime, Nat. Phys. 13, 39 (2017).

[30] J. Puertas Martinez, S. Leger, N. Gheeraert et al., A tunable Josephson platform to explore many-body quantum optics in circuit-QED, npj Quantum Inf. 5, 19 (2019).

[31] D. F. Walls and G. J. Milburn, Quantum Optics (Springer, Berlin, 1995).

[32] D. Manzano, A short introduction to the Lindblad master equation, AIP Advances 10, 025106 (2020).

[33] B. Peropadre, D. Zueco, D. Porras, and J. J. Garcia-Ripoll, Nonequilibrium and Nonperturbative Dynamics of Ultrastrong Coupling in Open Lines, Phys. Rev. Lett. 111, 243602 (2013).

[34] G. Diaz-Camacho, A. Bermudez, and J. J. Garcia-Ripoll, Dynamical polaron Ansatz: A theoretical tool for the ultrastrong-coupling regime of circuit QED, Phys. Rev. A 93, 043843 (2016).

[35] D. Zueco and J. Garcia-Ripoll, Ultrastrongly dissipative quantum Rabi model, Phys. Rev. A 99, 013807 (2019).

[36] W. S. Teixeira, F. L. Semiao, J. Tuorila, and M. Mottonen, Numerically exact treatment of dissipation in a driven two-level system, arXiv:2011.06106. 
[37] U. Fano, Effects of configuration interaction on intensities and phase shifts, Phys. Rev. 124, 1866 (1961).

[38] B. Huttner and S. M. Barnett, Quantization of the electromagnetic field in dielectrics, Phys. Rev. A 46, 4306 (1992).

[39] M. R. da Costa, A. O. Caldeira, S. M. Dutra, and H. Westfahl, Jr., Exact diagonalization of two quantum models for the damped harmonic oscillator, Phys. Rev. A 61, 022107 (2000).

[40] M. Bamba and T. Ogawa, Recipe for the Hamiltonian of systemenvironment coupling applicable to the ultrastrong-light-matterinteraction regime, Phys. Rev. A 89, 023817 (2014).
[41] The mode function is normalized so as to satisfy $\int_{0}^{\infty} d r f_{k}(r) f_{k^{\prime}}(r)=\delta\left(k-k^{\prime}\right)$.

[42] C. Ciuti and I. Carusotto, Input-output theory of cavities in the ultrastrong coupling regime: The case of timeindependent cavity parameters, Phys. Rev. A 74, 033811 (2006).

[43] M. Bamba and T. Ogawa, System-environment coupling derived by Maxwell's boundary conditions from the weak to the ultrastrong light-matter-coupling regime, Phys. Rev. A 88, 013814 (2013). 